\title{
Is metabolic syndrome a risk factor in hepatectomy? A meta-analysis with subgroup analysis for histologically confirmed hepatic manifestations
}

Anastasia Murtha-Lemekhova ${ }^{1}$, Juri Fuchs ${ }^{1}$, Svenja Feiler ${ }^{1}$, Erik Schulz ${ }^{1}$, Miriam Teroerde ${ }^{1}$, Eva Kalkum², Rosa Klotz ${ }^{1,2}$, Adrian Billeter ${ }^{1}$, Pascal Probst ${ }^{1,2,3}$ and Katrin Hoffmann ${ }^{1 *}$

\begin{abstract}
Background: Metabolic syndrome (MetS) is a risk factor in surgery. MetS can progress to metabolic (dysfunction)associated fatty liver disease (MAFLD), a vast-growing etiology of primary liver tumors which are major indications for liver surgery. The aim of this meta-analysis was to investigate the impact of MetS on complications and longterm outcomes after hepatectomy.

Methods: The protocol for this meta-analysis was registered at PROSPERO prior to data extraction. MEDLINE, Web of Science, and Cochrane Library were searched for publications on liver resections and MetS. Comparative studies were included. Outcomes encompassed postoperative complications, mortality, and long-term oncologic status. Data were pooled as odds ratio (OR) with a random-effects model. Risk of bias was assessed using the Quality in Prognostic Studies tool (QUIPS), and the certainty of the evidence was evaluated with GRADE. Subgroup analyses for patients with histopathologically confirmed non-alcoholic fatty liver disease (NAFLD) versus controls were performed.

Results: The meta-analyses included fifteen comparative studies. Patients with MetS suffered significantly more overall complications (OR 1.55; 95\% Cl $[1.05 ; 2.29] ; p=0.03$ ), major complications (OR 1.97 95\% Cl $[1.13 ; 3.43]$; $p=0.02$; $\left.P^{2}=62 \%\right)$, postoperative hemorrhages (OR 1.76; 95\% Cl $\left.[1.23 ; 2.50] ; p=0.01\right)$ and infections (OR 1.63; $95 \% \mathrm{Cl}[1.03$; 2.57]; $p=0.04)$. There were no significant differences in mortality, recurrence, 1- or 5-year overall or recurrence-free survivals. Patients with histologically confirmed NAFLD did not have significantly more overall complications; however, PHLF rates were increased (OR 4.87; 95\% CI [1.22; 19.47]; $p=0.04)$. Recurrence and survival outcomes did not differ significantly. The certainty of the evidence for each outcome ranged from low to very low.
\end{abstract}

\footnotetext{
* Correspondence: katrin.hoffmann@med.uni-heidelberg.de

'Department of General, Visceral, and Transplantation Surgery, Heidelberg University Hospital, Heidelberg, Germany

Full list of author information is available at the end of the article
}

(c) The Author(s). 2022 Open Access This article is licensed under a Creative Commons Attribution 4.0 International License, which permits use, sharing, adaptation, distribution and reproduction in any medium or format, as long as you give appropriate credit to the original author(s) and the source, provide a link to the Creative Commons licence, and indicate if changes were made. The images or other third party material in this article are included in the article's Creative Commons licence, unless indicated otherwise in a credit line to the material. If material is not included in the article's Creative Commons licence and your intended use is not permitted by statutory regulation or exceeds the permitted use, you will need to obtain permission directly from the copyright holder. To view a copy of this licence, visit http://creativecommons.org/licenses/by/4.0/ The Creative Commons Public Domain Dedication waiver (http://creativecommons.org/publicdomain/zero/1.0/) applies to the data made available in this article, unless otherwise stated in a credit line to the data. 
Conclusion: Patients with MetS that undergo liver surgery suffer more complications, such as postoperative hemorrhage and infection but not liver-specific complications-PHLF and biliary leakage. Histologically confirmed NAFLD is associated with significantly higher PHLF rates, yet, survivals of these patients are similar to patients without the MetS. Further studies should focus on identifying the tipping point for increased risk in patients with MetS-associated liver disease, as well as reliable markers of MAFLD stages and early markers of PHLF.

Trial registration: PROSPERO Nr: CRD42021253768

Keywords: Metabolic syndrome, liver surgery, post-hepatectomy liver failure, meta-analysis, NASH, NAFLD, MAFLD

\section{Background}

Metabolic syndrome (MetS) is a growing epidemic, marked by diabetes and obesity, that not only leads to increased rates of heart, vascular and gastrointestinal diseases, but presents a major risk factor in surgery $[1,2]$. In addition to increasing as the cause of hepatocellular carcinoma ( $\mathrm{HCC}), \mathrm{MetS}$ is associated with gastrointestinal and biliary tree cancers, thus being accountable for the rising indications in hepatobiliary surgery [3, 4]. Nonalcoholic fatty liver disease (NAFLD) and non-alcoholic steatohepatitis (NASH) are considered the hepatic manifestation of the MetS and are now summarized as the metabolic (dysfunction) associated fatty liver disease (MAFLD) [5]. This condition can lead to HCC without the conventional sequence of steatosis, steatohepatitis, fibrosis, cirrhosis, and, ultimately, HCC [6].

With indications for hepatobiliary surgery expanding, investigation of risk and predictive factors for posthepatectomy liver failure (PHLF), adequate postoperative liver regeneration, and other surgery-related outcomes intensifies in clinical importance [7, 8]. An accepted dogma of liver surgery dictates that for sufficient regeneration after hepatectomy, the future liver remnant (FLR) requires the volume of at least $20 \%$ from the initial organ. In case the liver parenchyma is altered due to chemotherapy or steatosis, e.g., due to MAFLD or alcohol overindulgence, at least $30 \%$ of the volume is required for the liver to sufficiently regenerate after resection [9].

As MetS is not only viewed as a patient-related risk factor for surgery but also as a risk factor for liverspecific complications in patients requiring liver surgery, a closer evaluation is warranted. The aim of this metaanalysis is to evaluate the MetS as a risk factor for posthepatectomy complications as well as a predictor of adverse oncological outcome.

\section{Methods}

The systematic review is reported according with the current PRISMA guidelines [10] and follows the recommendations of the Cochrane Handbook for Systematic Reviews and Interventions [11]. The protocol for this systematic review and meta-analysis was registered at PROSPERO prior to data extraction (CRD42021253768) [12].

The literature search was conducted in accordance with the recent recommendations [13]. The aim of the search was to identify all publications on liver resections in context of metabolic syndrome in humans. The search strategy is provided in Supplementary material. The searches were performed using MEDLINE via PubMed, Web of Science, and Cochrane Library. The last search was completed on August 27, 2021. Neither language nor publication date were restricted. Additionally, a hand search through references of included studies was performed.

Comparative study methodology was sought after with no restriction on prospective or retrospective design or blinding. Comments, editorials, meeting abstracts, correspondence, and reviews were excluded. The screening of titles and abstracts was performed by two independent reviewers (AML and JF). All disagreements were resolved through discussion and consultation with the third reviewer $(\mathrm{KH})$. Full-text review was performed independently by the same reviewers, after which all disagreements were once again resolved through discussion and consultation with the same third reviewer.

Data of included studies was extracted by two reviewers (AML and JF) independently using a standardized form composed prior to data extraction and adjusted based on the first two data extractions. Extracted data for each publication encompassed: title, authors, year of publication, country of publication, journal, source of funding, study methodology, cohorts' characteristics, interventions, and clinical outcomes (complication rates and gradings for post-hepatectomy liver failure, biliary leakage, bleeding, infections, ClavienDindo grades, mortality, recurrence, overall and recurrence-free survivals).

Meta-analyses were performed with $\mathrm{R}$ ( $\mathrm{R}$ version 4.0.3 using packages "metafor," "meta," and "ggplot2"). Forest plots present effect estimates. A random-effects model was utilized for all outcomes due to the heterogenic methodological and clinical framework of included studies [13]. Statistical heterogeneity was evaluated using the $I^{2}$ statistics. An $I^{2}$ value below $25 \%$ indicated low heterogeneity, while over 
Table 1 QUIPS

\begin{tabular}{lllllll}
\hline Study & $\begin{array}{l}\text { Study } \\
\text { participation }\end{array}$ & $\begin{array}{l}\text { Study } \\
\text { attrition }\end{array}$ & $\begin{array}{l}\text { Prognostic factor } \\
\text { measurement }\end{array}$ & $\begin{array}{l}\text { Outcome } \\
\text { measurement }\end{array}$ & $\begin{array}{l}\text { Study } \\
\text { confounding }\end{array}$ & $\begin{array}{l}\text { Statistical analysis and } \\
\text { reporting }\end{array}$ \\
\hline Koh 2019 & Moderate & High & Moderate & Low & Moderate & Moderate \\
Nishioka 2016 & Moderate & High & Low & Low & Moderate & Moderate \\
Billeter 2020 & Moderate & High & Moderate & Low & Moderate & Moderate \\
Hobeika 2019 & Moderate & Moderate & Moderate & Low & Moderate & Moderate \\
Bhazani 2012 & Moderate & High & Moderate & Low & Moderate & Moderate \\
Pais 2017 & Moderate & Moderate & Moderate & Low & Moderate & Moderate \\
Tian 2018 & Moderate & High & Moderate & Low & Low & Moderate \\
Tian 2020 & Moderate & High & High & Low & Low & Moderate \\
Yang 2020 & Moderate & High & Moderate & Low & Moderate & Moderate \\
Yoshida 2015 & Moderate & High & Moderate & Low & Migh & Moderate \\
Le Bien 2012 & Moderate & High & Moderate & Low & Moderate & Moderate \\
Jung 2020 & Moderate & High & Moderate & Low & Moderate & Moderate \\
Wakai 2011 & Moderate & High & Moderate & Low & Moderate & Moderate \\
Conci 2021 & Moderate & Moderate & Low & Low & Moderate & Moderate \\
Fagenson & Moderate & Moderate & Moderate & & &
\end{tabular}

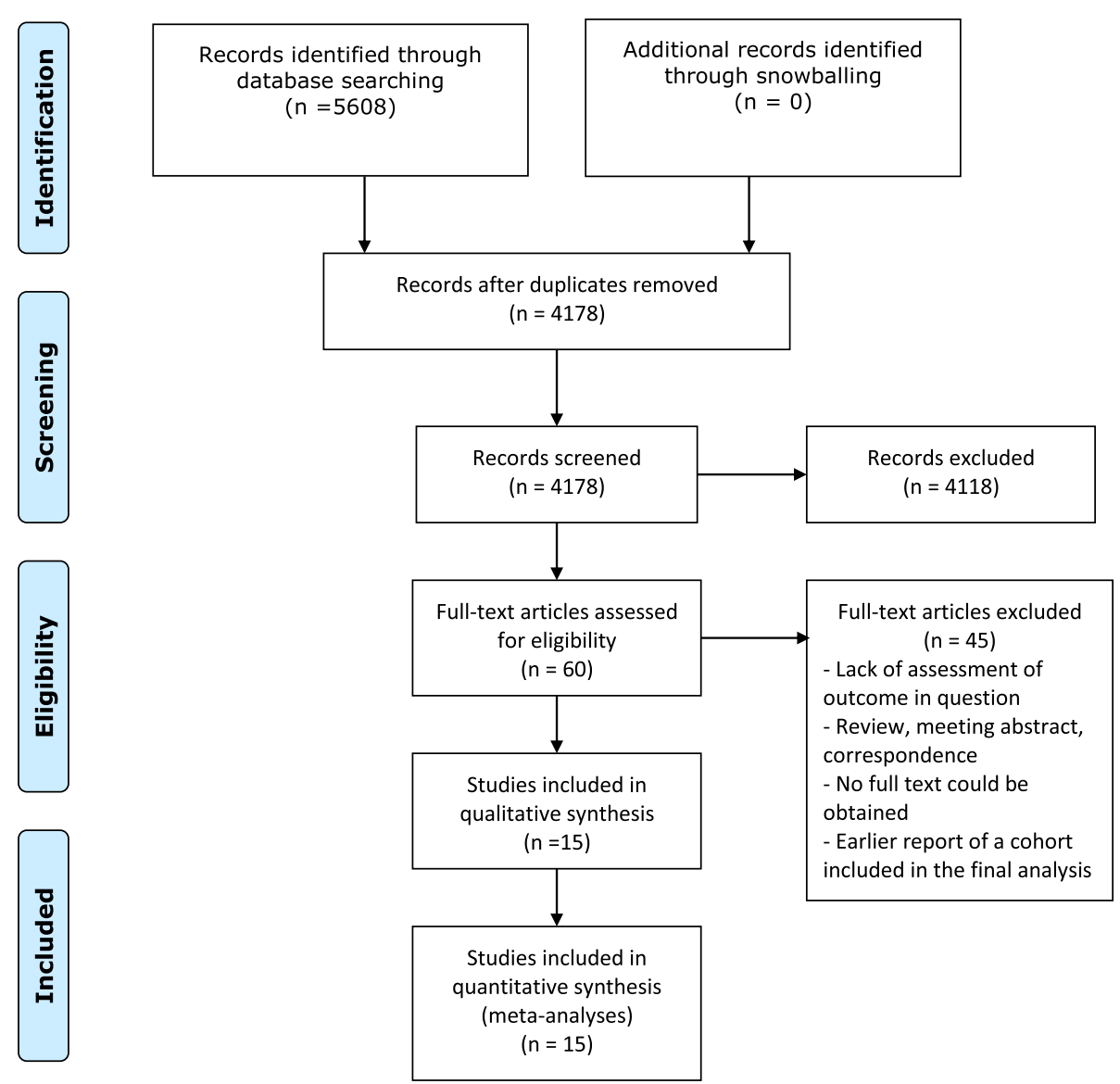

Fig. 1 Flowchart of the selection process. The flow diagram depicts the steps of the systematic review process 
Table 2 Overview of included publications

\begin{tabular}{|c|c|c|c|c|c|c|c|c|c|}
\hline \multirow[t]{2}{*}{ Publication } & \multirow[t]{2}{*}{ Methodology } & \multirow[t]{2}{*}{ Definition of MetS/NAFLD used } & \multirow{2}{*}{$\begin{array}{l}\text { Included in } \\
\text { subgroup } \\
\text { analysis for } \\
\text { histologically } \\
\text { proven NAFL } \\
\text { D }\end{array}$} & \multicolumn{2}{|c|}{ Cohorts } & \multicolumn{2}{|l|}{ Indications } & \multicolumn{2}{|c|}{ Hepatectomy } \\
\hline & & & & $\begin{array}{l}\text { MetS } \\
(n)\end{array}$ & $\begin{array}{l}\text { Control } \\
(n)\end{array}$ & MetS $n(\%)$ & $\begin{array}{l}\text { Control } n \\
(\%)\end{array}$ & $\begin{array}{l}\text { MetS } \\
n(\%)\end{array}$ & $\begin{array}{l}\text { Control } \\
n(\%)\end{array}$ \\
\hline Koh 2019 & $\begin{array}{l}\text { Retrospective, } \\
\text { single center }\end{array}$ & NAFLD: NASH Clinical Research Network criteria [32] & Yes & 152 & 844 & HCC 152 & HCC 844 & $\begin{array}{l}\text { Major } \\
21 \\
(14 \%) \\
\text { Minor } \\
131 \\
(86 \%)\end{array}$ & $\begin{array}{l}\text { Major } \\
238 \\
(28 \%) \\
\text { Minor } \\
606 \\
(75 \%)\end{array}$ \\
\hline $\begin{array}{l}\text { Vigano } \\
2015\end{array}$ & $\begin{array}{l}\text { Retrospective, } \\
\text { multicenter, } \\
\text { matched }\end{array}$ & $\begin{array}{l}\text { MetS: National Cholesterol Education Program's Adult } \\
\text { Treatment Panel III report [33] }\end{array}$ & No & 96 & 96 & HCC 96 & HCC 96 & $\begin{array}{l}\text { Major } \\
41 \\
(43 \%) \\
\text { Minor } \\
55 \\
(57 \%)\end{array}$ & $\begin{array}{l}\text { Major } 41 \\
(43 \%) \\
\text { Minor } \\
55(57 \%)\end{array}$ \\
\hline $\begin{array}{l}\text { Nishioka } \\
2016\end{array}$ & $\begin{array}{l}\text { Retrospective, } \\
\text { single center }\end{array}$ & MetS: diabetes, HT and dyslipidemia & No & 14 & 15 & ihCC 14 & ihCC 13 & $\begin{array}{l}\text { Major } \\
13 \\
(93 \%) \\
\text { Minor } \\
1(7 \%)\end{array}$ & $\begin{array}{l}\text { Major } 13 \\
(100 \%) \\
\text { Minor } 0\end{array}$ \\
\hline $\begin{array}{l}\text { Billeter } \\
2020\end{array}$ & $\begin{array}{l}\text { Retrospective, } \\
\text { single center, } \\
\text { matched }\end{array}$ & NAFLD: SAF score [34] & Yes & 34 & 54 & HCC 34 & HCC 54 & $\begin{array}{l}\text { Major } \\
19 \\
(56 \%) \\
\text { Minor } \\
15 \\
(44 \%)\end{array}$ & $\begin{array}{l}\text { Major } 7 \\
(13 \%) \\
\text { Minor } \\
47(87 \%)\end{array}$ \\
\hline $\begin{array}{l}\text { Hobeika } \\
2019\end{array}$ & $\begin{array}{l}\text { Retrospective, } \\
\text { single center }\end{array}$ & $\begin{array}{l}\text { MetS: } \geq 3 \text { criteria_central obesity, dyslipidemia, DM2/ } \\
\text { glucose intolerance, HT. NAFLD: NASH Clinical Research } \\
\text { Network Scoring System [32] }\end{array}$ & Yes & 40 & 75 & ihCC 40 & ihCC 75 & $\begin{array}{l}\text { Major } \\
28 \\
(70 \%) \\
\text { Minor } \\
12 \\
(30 \%)\end{array}$ & $\begin{array}{l}\text { Major } 63 \\
(84 \%) \\
\text { Minor } \\
12(16 \%)\end{array}$ \\
\hline $\begin{array}{l}\text { Bhayani } \\
2012\end{array}$ & $\begin{array}{l}\text { Retrospective, } \\
\text { database }\end{array}$ & MetS: $\mathrm{BMI} \geq 30 \mathrm{~kg} / \mathrm{m}^{2}$, diabetes and $\mathrm{HT}$ & No & 256 & 3717 & $\begin{array}{l}\text { Metastasis } \\
113(44 \%) \\
\text { HCC } 75 \\
(29 \%) \\
\text { ihCC 9 (4\%) } \\
\text { Other } \\
\text { malignancy } \\
18 \text { (7\%) } \\
\text { Benign } \\
\text { lesions } 14 \\
(5 \%)\end{array}$ & $\begin{array}{l}\text { Metastasis } \\
1717(46 \%) \\
\text { HCC } 596 \\
(16 \%) \\
\text { ihCC } 130 \\
(4 \%) \\
\text { Other } \\
\text { malignancy } \\
253 \text { (7\%) } \\
\text { Benign } \\
\text { lesions } 441 \\
(7 \%)\end{array}$ & $\begin{array}{l}\text { Major } \\
86 \\
(34 \%) \\
\text { Minor } \\
170 \\
(66 \%)\end{array}$ & $\begin{array}{l}\text { Major } \\
1546 \\
(42 \%) \\
\text { Minor } \\
2171 \\
(58 \%)\end{array}$ \\
\hline Pais 2017 & $\begin{array}{l}\text { Retrospective, } \\
\text { multicenter }\end{array}$ & $\begin{array}{l}\text { NAFLD: metabolic risk factors (overweight or obesity } \\
\text { defined as BMI } \geq 25 \mathrm{~kg} / \mathrm{m}^{2}, \mathrm{DM} 2, \mathrm{HT} \text {, dyslipidemia), and } \\
\text { absence of other causes of liver disease }\end{array}$ & No & 39 & 284 & HCC 39 & HCC 284 & $\begin{array}{l}\text { Major } \\
19 \\
(49 \%) \\
\text { Minor } \\
20 \\
(51 \%)\end{array}$ & $\begin{array}{l}\text { Major } \\
108 \\
(38 \%) \\
\text { Minor } \\
176 \\
(62 \%)\end{array}$ \\
\hline Tian 2018 & $\begin{array}{l}\text { Retrospective, } \\
\text { single center }\end{array}$ & $\begin{array}{l}\text { MetS: central obesity (waist circumference } \geq 90 \mathrm{~cm} \text { for } \\
\text { men, } \geq 80 \mathrm{~cm} \text { for women) plus } 2 \text { of the following: } \\
\text { elevated triglycerides ( } \geq 150 \mathrm{mg} / \mathrm{dL} \text { or specific } \\
\text { treatment), reduced } \mathrm{HDL} \text { cholesterol }(<40 \mathrm{mg} / \mathrm{dL} \text { in } \\
\text { males and }<50 \mathrm{mg} / \mathrm{dL} \text { in females or specific } \\
\text { treatment), elevated BP (systolic BP } \geq 130 \text { or diastolic } \\
\mathrm{BP} \geq 85 \mathrm{mmH} \text { or treatment for } \mathrm{HT}) \text {, elevated fasting } \\
\text { plasma glucose } \geq 100 \mathrm{mg} / \mathrm{dL} \text { or } \mathrm{DM} 2\end{array}$ & No & 81 & 1154 & HCC 81 & HCC 1154 & $\begin{array}{l}\text { Major } \\
9 \\
(11 \%) \\
\text { Minor } \\
72 \\
(89 \%)\end{array}$ & $\begin{array}{l}\text { Major } \\
204 \\
(18 \%) \\
\text { Minor } \\
950 \\
(92 \%)\end{array}$ \\
\hline Tian 2020 & $\begin{array}{l}\text { Retrospective, } \\
\text { single center, } \\
\text { matched }\end{array}$ & $\begin{array}{l}\text { MetS: central obesity (waist circumference with } \\
\text { ethnicity-specific values) and any two: reduced } \mathrm{HDL} \text { (< } \\
40 \mathrm{mg} / \mathrm{dL} \text { in males and }<50 \mathrm{mg} / \mathrm{dL} \text { in females or spe- } \\
\text { cific treatment), elevated triglycerides }(\geq 150 \mathrm{mg} / \mathrm{dL} \text { or } \\
\text { specific treatment), elevated fasting plasma glucose } \geq \\
100 \mathrm{mg} / \mathrm{dL} \text { or DM2, elevated BP (systolic BP } \geq 130 \text { or } \\
\text { diastolic BP } \geq 85 \mathrm{mmHg} \text { or treatment for HT) }\end{array}$ & No & 74 & 74 & HCC 74 & HCC 74 & $\begin{array}{l}\text { Major } \\
4(5 \%) \\
\text { Minor } \\
70 \\
(95 \%)\end{array}$ & $\begin{array}{l}\text { Major } 2 \\
(3 \%) \\
\text { Minor } \\
72(97 \%)\end{array}$ \\
\hline Yang 2020 & $\begin{array}{l}\text { Retrospective, } \\
\text { multicenter, } \\
\text { matched }\end{array}$ & $\begin{array}{l}\text { NAFLD is defined as MetS (overweight or obesity, DM2, } \\
\text { HT, and dyslipidemia), consistent US features of fatty } \\
\text { liver, and/or past or present histological features of } \\
\text { hepatic fatty infiltration with an alcohol intake }<30 \mathrm{~g} /\end{array}$ & No & 89 & 89 & HCC 89 & HCC 89 & $\begin{array}{l}\text { Major } \\
32 \\
(36 \%) \\
\text { Minor }\end{array}$ & $\begin{array}{l}\text { Major } 27 \\
(30 \%) \\
\text { Minor } \\
62(70 \%)\end{array}$ \\
\hline
\end{tabular}


Table 2 Overview of included publications (Continued)

\begin{tabular}{|c|c|c|c|c|c|c|c|c|c|}
\hline \multirow[t]{2}{*}{ Publication } & \multirow[t]{2}{*}{ Methodology } & \multirow[t]{2}{*}{ Definition of MetS/NAFLD used } & \multirow{2}{*}{$\begin{array}{l}\text { Included in } \\
\text { subgroup } \\
\text { analysis for } \\
\text { histologically } \\
\text { proven NAFL } \\
\text { D }\end{array}$} & \multicolumn{2}{|c|}{ Cohorts } & \multicolumn{2}{|l|}{ Indications } & \multicolumn{2}{|c|}{ Hepatectomy } \\
\hline & & & & $\begin{array}{l}\text { MetS } \\
(n)\end{array}$ & $\begin{array}{l}\text { Control } \\
\text { (n) }\end{array}$ & MetS $n(\%)$ & $\begin{array}{l}\text { Control } n \\
(\%)\end{array}$ & $\begin{array}{l}\text { MetS } \\
n(\%)\end{array}$ & $\begin{array}{l}\text { Control } \\
n(\%)\end{array}$ \\
\hline & & day & & & & & & $\begin{array}{l}57 \\
(64 \%)\end{array}$ & \\
\hline $\begin{array}{l}\text { Yoshida } \\
2015\end{array}$ & $\begin{array}{l}\text { Retrospective, } \\
\text { single center }\end{array}$ & $\begin{array}{l}\text { MetS: } \geq 3 \text { of the following: central obesity (waist } \\
\text { circumference } \geq 90 \mathrm{~cm} \text { in men and } \geq 80 \mathrm{~cm} \text { in women, } \\
\mathrm{BMI} \geq 28 \mathrm{~kg} / \mathrm{m} 2, \text { dyslipidemia (triglycerides } \geq 150 \mathrm{mg} / \\
\mathrm{dL} \text { or } \mathrm{HDL} \geq 40 \mathrm{mg} / \mathrm{dL} \text { in men, } \geq 50 \mathrm{mg} / \mathrm{dL} \text { in women), } \\
\mathrm{HT} \text { (BP } \geq 130 / 85 \mathrm{mmHg} \text { ), and diabetes mellitus (fasting } \\
\text { glucose }[100 \mathrm{mg} / \mathrm{dL} \text { ) }\end{array}$ & No & 35 & 114 & HCC 35 & HCC 114 & $\begin{array}{l}\text { Major } \\
3(9 \%) \\
\text { Minor } \\
32 \\
(91 \%)\end{array}$ & $\begin{array}{l}\text { Major } 8 \\
(7 \%) \\
\text { Minor } \\
106 \\
(93 \%)\end{array}$ \\
\hline $\begin{array}{l}\text { Le Bian } \\
2012\end{array}$ & $\begin{array}{l}\text { Retrospective, } \\
\text { single center }\end{array}$ & $\begin{array}{l}\text { MetS: } \geq 3 \text { of the following: overweight or obesity } \\
\left.\text { (BMI }>25 \mathrm{~kg} / \mathrm{m}^{2}\right) \text {, DM (defined as fasting plasma } \\
\text { glucose }>5.5 \mathrm{mmol} / \mathrm{L} \text { ), HT (BP }>130 / 85 \mathrm{mmHg} \text { ), and } \\
\text { dyslipidemia (triglycerides } \geq 1.7 \mathrm{mmol} / \mathrm{l} \text { and } / \mathrm{or} \mathrm{HDL}<1 \\
\mathrm{mmol} / \mathrm{L} \text { in males or }<1.3 \mathrm{mmol} / \mathrm{L} \text { in females). }\end{array}$ & No & 30 & 121 & $\begin{array}{l}\text { Primary } 10 \\
(33 \%) \\
\text { Metastasis } \\
17(57 \%) \\
\text { Benign } 3 \\
(10 \%)\end{array}$ & $\begin{array}{l}\text { Primary } 29 \\
(24 \%) \\
\text { Metastasis } \\
59(49 \%) \\
\text { Benign } 33 \\
(27 \%)\end{array}$ & $\begin{array}{l}\text { Major } \\
30\end{array}$ & $\begin{array}{l}\text { Major } \\
121\end{array}$ \\
\hline Jung 2020 & $\begin{array}{l}\text { Retrospective, } \\
\text { single center, } \\
\text { matched }\end{array}$ & $\begin{array}{l}\text { NAFLD criteria of the American Association for the } \\
\text { Study of Liver Disease [33] }\end{array}$ & Yes & 32 & 32 & HCC 32 & HCC 32 & $\begin{array}{l}\text { Major } \\
24 \\
(75 \%) \\
\text { Minor } \\
8 \\
(25 \%)\end{array}$ & $\begin{array}{l}\text { Major } 18 \\
(56 \%) \\
\text { Minor } \\
14(44 \%)\end{array}$ \\
\hline Wakai 2011 & $\begin{array}{l}\text { Retrospective, } \\
\text { single center }\end{array}$ & NAFLD: NASH Clinical Research Network criteria [32] & Yes & 17 & 208 & HCC 17 & HCC 208 & $\begin{array}{l}\text { Major } \\
8 \\
(47 \%) \\
\text { Minor } \\
9 \\
(53 \%)\end{array}$ & $\begin{array}{l}\text { Major } 59 \\
(28 \%) \\
\text { Minor } \\
149 \\
(72 \%)\end{array}$ \\
\hline Conci 2021 & $\begin{array}{l}\text { Retrospective, } \\
\text { multicenter, } \\
\text { matched }\end{array}$ & $\begin{array}{l}\text { MAFLD: hepatic steatosis (imaging, blood scores/ } \\
\text { markers, or histology) and overweight (BMI } \geq 25 \mathrm{~kg} / \mathrm{m}^{2} \\
\text { in Caucasians or } \geq 23 \text { in Asians) or DM2 or normal } \\
\text { weight in the presence of two or more metabolic } \\
\text { abnormalities (high waist circumference, HT, elevated } \\
\text { triglycerides or cholesterol, insulin resistance or } \\
\text { prediabetes, and high level of plasma c-reactive } \\
\text { protein). }\end{array}$ & No & 85 & 255 & HCC 85 & HCC 255 & $\begin{array}{l}\text { Major } \\
18 \\
(21 \%) \\
\text { Minor } \\
67 \\
(79 \%)\end{array}$ & $\begin{array}{l}\text { Major } 44 \\
(17 \%) \\
\text { Minor } \\
211 \\
(83 \%)\end{array}$ \\
\hline $\begin{array}{l}\text { Fagenson } \\
2020\end{array}$ & $\begin{array}{l}\text { Retrospective, } \\
\text { database, } \\
\text { matched }\end{array}$ & $\begin{array}{l}\text { MetS: } 3 \text { elements obesity (BMI }>30 \mathrm{~kg} / \mathrm{m} 2, \mathrm{HT} \text {, and } \\
\text { diabetes mellitus) }\end{array}$ & No & 863 & 863 & $\begin{array}{l}\text { Primary } 261 \\
(30 \%) \\
\text { Metastasis } \\
457(53 \%) \\
\text { Benign } 145 \\
(17 \%)\end{array}$ & $\begin{array}{l}\text { Primary } 258 \\
(30 \%) \\
\text { Metastasis } \\
462(53 \%) \\
\text { Benign } 143 \\
(17 \%)\end{array}$ & $\begin{array}{l}\text { Major } \\
863\end{array}$ & $\begin{array}{l}\text { Major } \\
863\end{array}$ \\
\hline
\end{tabular}

BP blood pressure, DM2 diabetes mellitus type 2, HCC hepatocellular carcinoma, $H D L$ high-density lipoprotein cholesterol, $H T$ hypertension, $M A F L D$ metabolically associated fatty liver disease, MetS metabolic syndrome, NAFLD non-alcoholic fatty liver disease, NASH non-alcoholic steatohepatitis US ultrasound

$75 \%$ was considered high. Odds ratios and 95\% confidence intervals for dichotomous endpoints were pooled with the Mantel-Haenszel method

The methodological quality of included studies was performed using QUIPS [14]. Detailed information about assessed qualities is provided in Table 1. The certainty of the evidence was assessed using GRADE $[15,16]$.

Based on the result of the full-text examination, the following endpoints could be evaluated in the quantitative analysis: overall complications, minor and major complications, PHLF, postoperative infections, postoperative biliary leakage and hemorrhage, perioperative and 90-day mortalities, recurrence, 1- and 5-year overall survivals (OS), and 1- and 5-year recurrencefree survivals (RFS).

\section{Results}

The search identified 4178 potentially relevant publications after exclusion of duplicates. After screening of titles and abstracts, 60 full texts were further evaluated for inclusion. Inclusion criteria were fulfilled by fifteen articles which were then included into qualitative and quantitative analyses (Fig. 1). Although most studies described patients with HCC [17-26], two studies [27, 28] reported on patients undergoing hepatectomy for intrahepatic cholangiocarcinoma and another three on patients with various benign and malignant liver tumors [29-31]. Table 2 provides an overview of core data extracted from each study. Only two studies described patients undergoing major hepatectomy while others had predominantly minor hepatectomy cohorts [30,31]. Due to different endpoints used by both studies, no 


\begin{tabular}{|c|c|c|c|c|c|c|c|c|c|}
\hline Study & Events & $\begin{array}{l}\text { MetS } \\
\text { Total }\end{array}$ & \multicolumn{2}{|c|}{ Control } & \multicolumn{2}{|r|}{ Odds Ratio } & OR & $95 \%-\mathrm{Cl}$ & Weight \\
\hline Koh 2019 & 85 & 152 & 260 & 844 & & +1 & 2.85 & {$[2.00 ; 4.05]$} & $11.9 \%$ \\
\hline Nishioka 2016 & 8 & 14 & 2 & 13 & & $\mp$ & 7.33 & {$[1.16 ; 46.23]$} & $3.2 \%$ \\
\hline Billeter 2020 & 19 & 34 & 31 & 54 & & & 0.94 & {$[0.40 ; 2.23]$} & $7.8 \%$ \\
\hline Hobeika 2019 & 33 & 40 & 59 & 75 & & & 1.28 & {$[0.48 ; 3.42]$} & $6.9 \%$ \\
\hline Bhayani 2012 & 73 & 256 & 846 & 3717 & & & 1.35 & {$[1.02 ; 1.79]$} & $12.4 \%$ \\
\hline Tian 2018 & 35 & 81 & 450 & 1154 & & & 1.19 & {$[0.76 ; 1.88]$} & $11.1 \%$ \\
\hline Tian 2020 & 23 & 74 & 9 & 74 & & 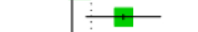 & 3.26 & {$[1.39 ; 7.65]$} & $7.9 \%$ \\
\hline Yoshida 2015 & 14 & 35 & 47 & 114 & & & 0.95 & {$[0.44 ; 2.06]$} & $8.5 \%$ \\
\hline Wakai 2011 & 10 & 17 & 62 & 208 & & 1 & 3.36 & {$[1.22 ; 9.24]$} & $6.8 \%$ \\
\hline Conci 2021 & 35 & 85 & 118 & 255 & & & 0.81 & {$[0.49 ; 1.34]$} & $10.8 \%$ \\
\hline Fagenson 2020 & 323 & 863 & 273 & 863 & & & 1.29 & {$[1.06 ; 1.58]$} & $12.8 \%$ \\
\hline Overall effect & & 1651 & & 7371 & & & 1.55 & {$[1.05 ; 2.29]$} & $100.0 \%$ \\
\hline Heterogeneity: $I^{2}$ & $\%, \tau^{2}=0$ & $.2759, p$ & $p<0.01$ & & $\Gamma$ & \begin{tabular}{l|l|l}
1 & 1 & 1
\end{tabular} & & & \\
\hline Test for overall eff & $t_{10}=2.50$ & $(p=0$. & .03) & & 0.1 & 0.512 & & & \\
\hline
\end{tabular}

b

Forest plot for major complications in MetS patients versus control after hepatectomy

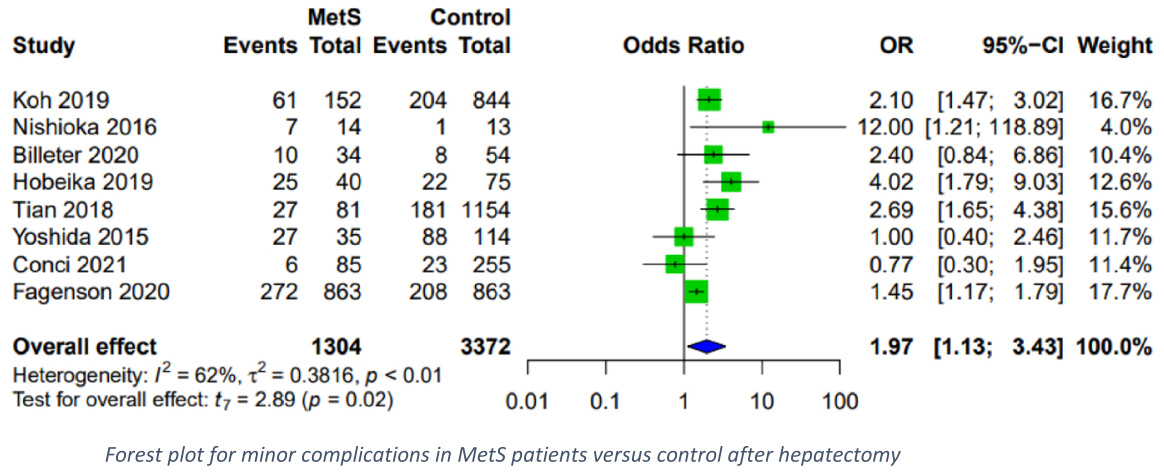

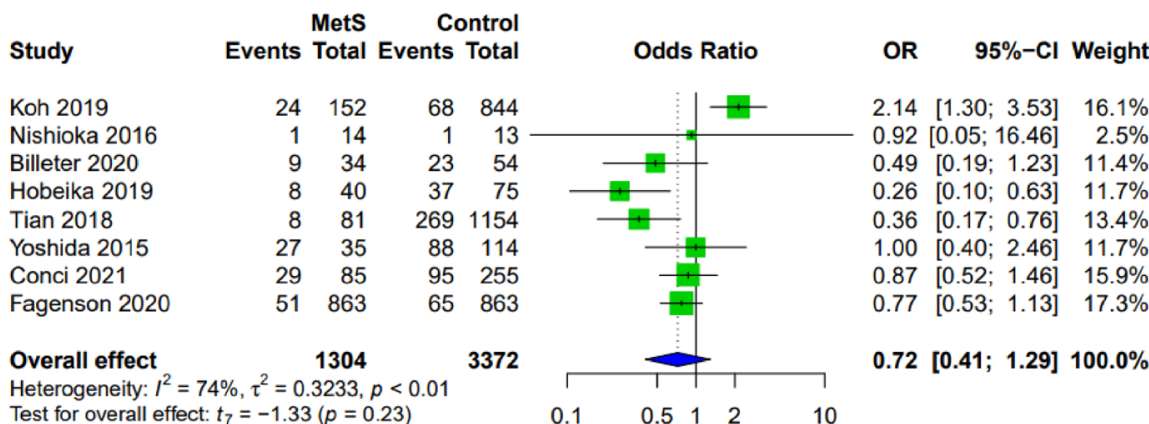

Fig. 2 Forest plots for overall, major and minor complications in MetS patients versus control after hepatectomy. A random-effects model was utilized for all outcomes due to heterogenic methodological and clinical framework of included studies. Statistical heterogeneity was evaluated using the $P^{2}$ statistics. An $P^{2}$ value below 25\% indicated low heterogeneity, while over 75\% was considered high

quantitative analysis could be performed for major hepatectomy cohorts.

\section{Critical appraisal of included studies}

The risk of bias assessment was performed using the tool for Quality in Prognostic Studies (QUIPS) and included assessment of six main domains: study participation, study attrition, prognostic factor measurement, outcome measurement, study confounding, and statistical analysis and reporting. Table 1 provides an overview of the assessment. Overall, included studies had a moderate risk of bias.

\section{Complications}

Overall complications were assessed based on data from eleven studies [17, 19, 21-23, 25-29, 31]. Data on major and minor complications was available in eight $[17,19$, 22, 25-28, 31]. Overall, patients with the MetS suffered significantly more overall complications compared to control. There was also a significant increase in major complications for patients with MetS (Fig. 2). 


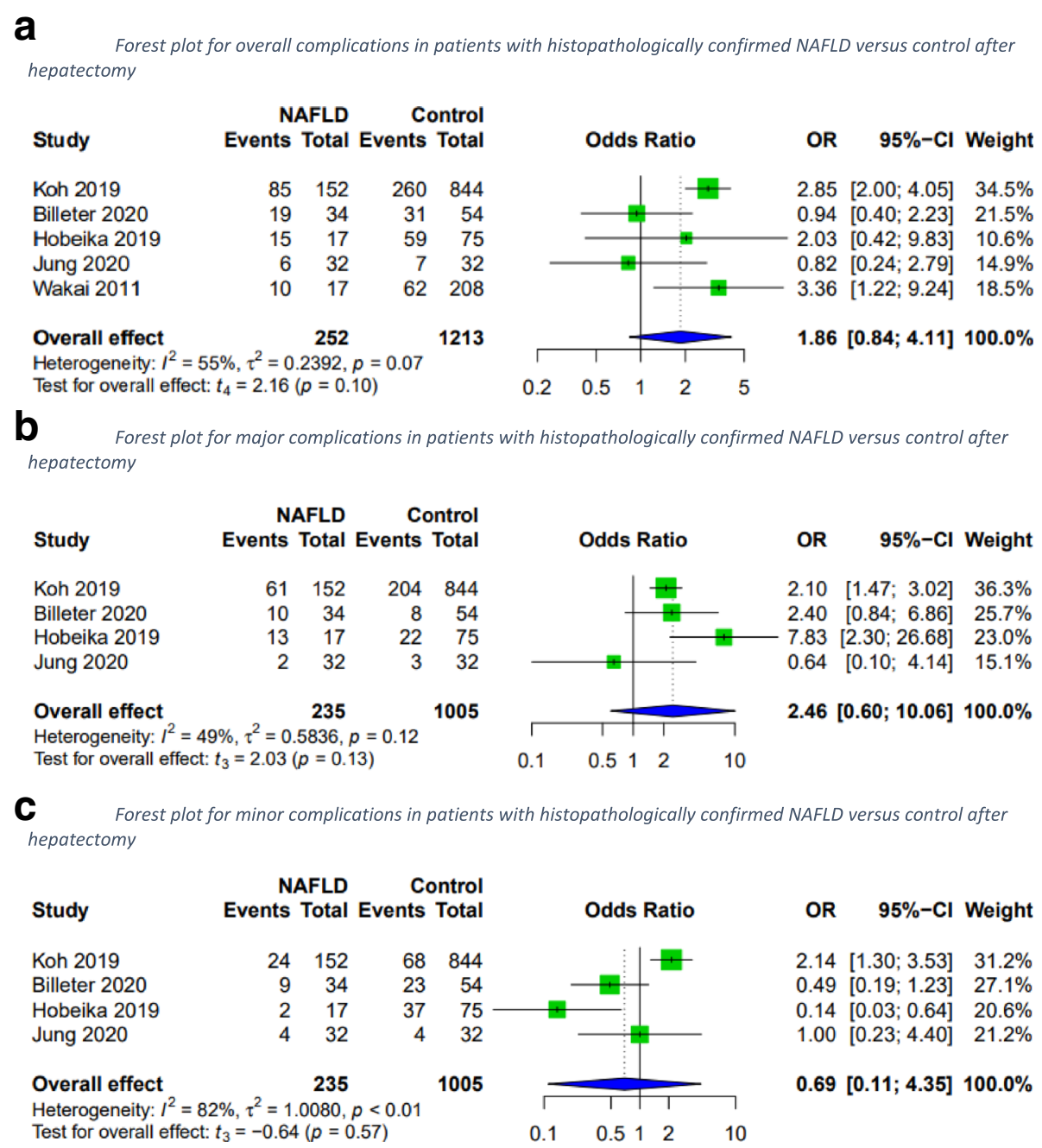

Fig. 3 Forest plots for overall, major and minor complications in patients with histopathologically confirmed NAFLD versus control after hepatectomy. A random-effects model was utilized for all outcomes due to the heterogenic methodological and clinical framework of included studies. Statistical heterogeneity was evaluated using the $P^{2}$ statistics. An $P^{2}$ value below $25 \%$ indicated low heterogeneity, while over $75 \%$ was considered high

In a subgroup analysis of patients with histopathologically confirmed NAFLD versus control, the two groups did not vary significantly in overall complications. No difference was observed for major or minor complications either (Fig. 3).

\section{Liver-specific complications}

Liver-specific complications after hepatectomy for patients with the MetS versus those without were assessed. PHLF rates, biliary leakages, and postoperative infections did not differ between groups; however, postoperative hemorrhage occurred significantly more frequently in MetS patients (Fig. 4).

In patients with histopathologically confirmed NAFLD, PHLF occurred more frequently than in the control group (Fig. 5), while postoperative biliary leakage, infection and hemorrhage were comparable to the control group (OR 1.21; 95 CI [0.01; 178.13]; $p=0.88 ; I^{2}=58 \%$, OR $2.17 ; 95 \%$ CI $[0.00 ; 5413.25] ; p=0.43 ; I^{2}=74 \%$, and OR $2.08 ; 95 \%$ CI $\left.[0.49 ; 8.85] ; p=0.16 ; I^{2}=0 \%\right)$. Postoperative biliary leakage and hemorrhage were reported in three studies, while only two provided data on postoperative infections.

\section{Mortality}

Perioperative mortality was assessed in two studies, which showed similar results in MetS patients and the control groups (OR 4.84; 95\% CI [0.00; 41157788.03]; $p=$ $\left.0.43 ; I^{2}=62 \%\right)$. Ninety-day mortality was reported in five studies and showed only a minor difference in patients with and without MetS (Fig. 6).

Ninety-day mortality did not show a difference in the subgroup analysis for patients with histopathologically 
a

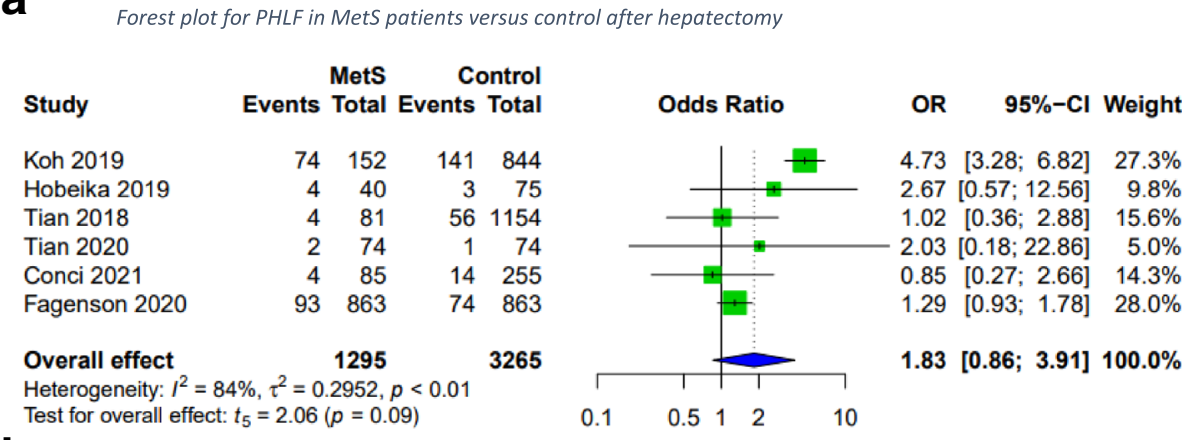

b Forest plot for postoperative biliary leakage in MetS patients versus control after hepatectomy

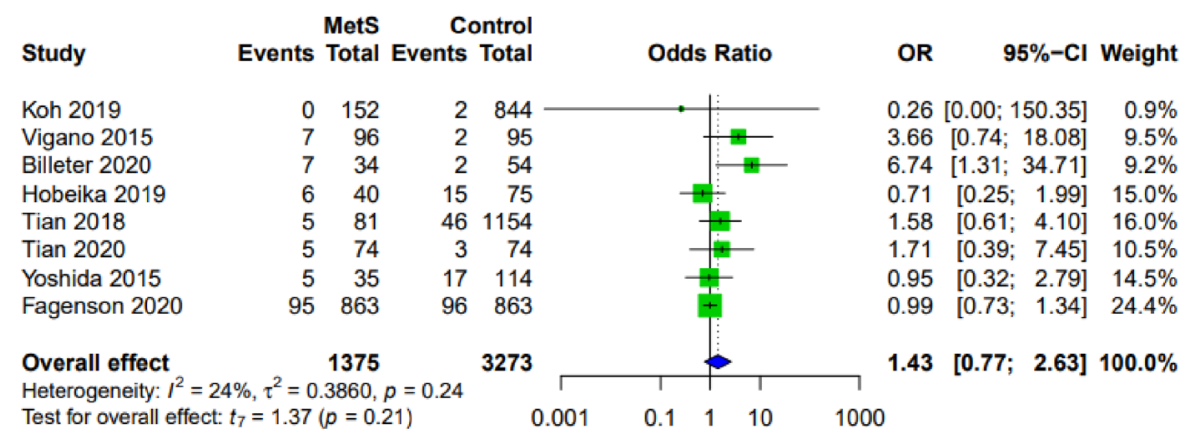

C Test for overall effect: $t_{7}=1.37(p=0.21)$
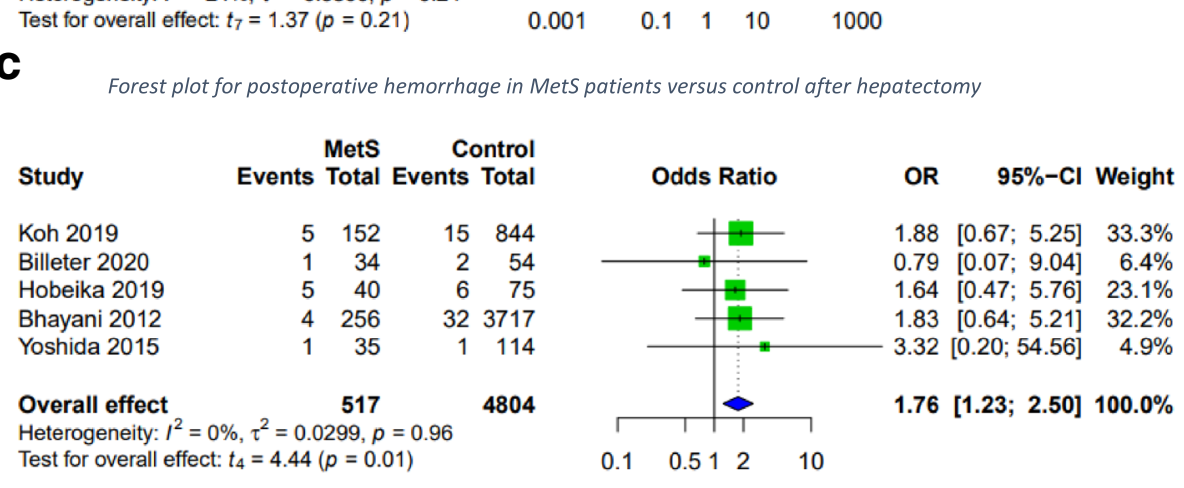

d Forest plot for postoperative infections in Mets patients versus control after hepatectomy

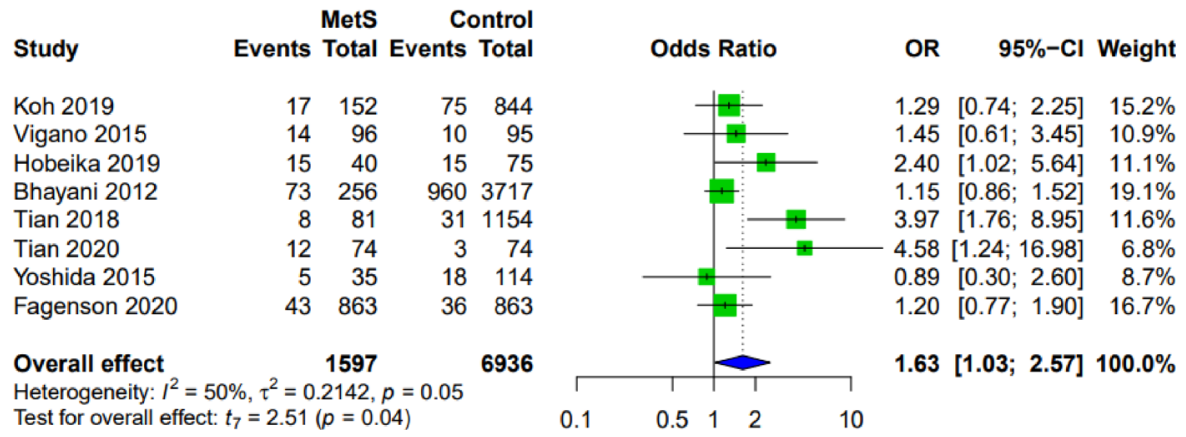

Fig. 4 Forest plots for liver-specific complications in MetS patients versus control after hepatectomy. A random-effects model was utilized for all outcomes due to the heterogenic methodological and clinical framework of included studies. Statistical heterogeneity was evaluated using the 12 statistics. An $P^{2}$ value below $25 \%$ indicated low heterogeneity, while over $75 \%$ was considered high 


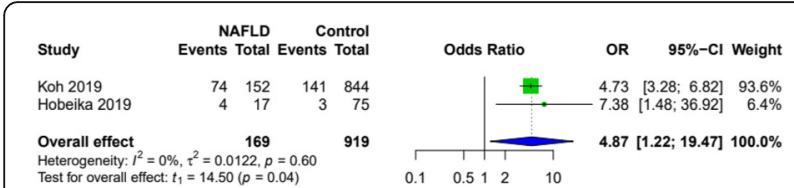

Fig. 5 Forest plot for PHLF in patients with histopathologically confirmed NAFLD versus control after hepatectomy. A randomeffects model was utilized for all outcomes due to the heterogenic methodological and clinical framework of included studies. Statistical heterogeneity was evaluated using the $P^{2}$ statistics. An $P^{2}$ value below $25 \%$ indicated low heterogeneity, while over $75 \%$ was considered high

confirmed NAFLD versus control (OR 1.90; 95\% CI $\left.[0.00 ; 179526.98] ; p=0.61 ; I^{2}=74 \%\right)$. However, only two studies reported this outcome. Perioperative mortality could not be analyzed for the subgroup due to insufficient data.

\section{Oncological outcomes}

Recurrence rates were similar in patients with and without the MetS. Three studies provided sufficient data for the outcome (Fig. 7).

For patients with histopathologically confirmed NAFL $\mathrm{D}$ versus control, recurrence rates did not show significant differences (OR 1.11; 95\% CI [0.69; 1.79]; $p=0.43$; $\left.I^{2}=0 \%\right)$. However, only three studies provided data for this endpoint.

One-year OS was reported in five publications and did not show significant differences between groups (OR 0.83 ; 95\% CI [0.32; 2.14]; $\left.p=0.61 ; I^{2}=39 \%\right)$. Six publications described 5-year OS rates, which did not show a significant difference between groups (OR 1.07; 95\% CI $\left.[0.79 ; 1.45] ; p=0.58 ; I^{2}=8 \%\right)$. These differences were also insignificant in patients with histopathologically confirmed NAFLD versus control: 1-year OS OR 0.79; 95\% [0.00; 93283.03]; $p=0.84 ; I^{2}=80 \%, 5$-year OS OR 1.39 $[0.85 ; 2.26] ; p=0.10 ; I^{2}=0 \%$. These overall effects estimated were based on data from two and three studies, respectively.

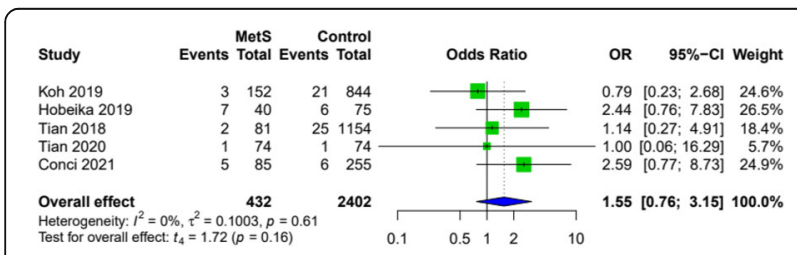

Fig. 6 Forest plot for 90-day mortality in MetS patients versus control after hepatectomy. A random-effects model was utilized for all outcomes due to the heterogenic methodological and clinical framework of included studies. Statistical heterogeneity was evaluated using the $R^{2}$ statistics. An $R^{2}$ value below 25\% indicated low heterogeneity, while over $75 \%$ was considered high

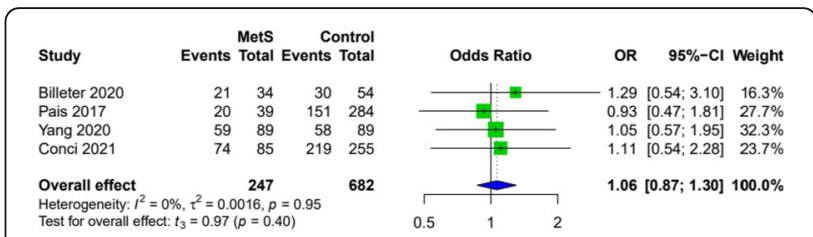

Fig. 7 Forest plot for tumor recurrence in MetS patients versus control after hepatectomy. A random-effects model was utilized for all outcomes due to the heterogenic methodological and clinical framework of included studies. Statistical heterogeneity was evaluated using the $P^{2}$ statistics. An $P^{2}$ value below 25\% indicated low heterogeneity, while over $75 \%$ was considered high

One-year RFS was similar in patients with and without the MetS (OR 0.95; 95\% CI $[0.47 ; 1.92] ; p=0.84 ; I^{2}=0 \%$ ), as was the 5 -year RFS (OR 1.20; 95\% CI $[0.91 ; 1.59]$; $p=$ $\left.0.14 ; I^{2}=0 \%\right)$. These were also the effects seen in patients with histopathologically confirmed NAFLD versus control (1-year RFS OR 0.96; 95\% CI [0.00; 920.79]; $p=0.95$; $I^{2}=19 \%$ and 5 -year RFS OR 1.23 ; 95\% CI $[0.76 ; 1.99] ; p=$ $\left.0.21 ; I^{2}=0 \%\right)$

\section{Certainty of evidence (GRADE)}

A rating of certainty of evidence was made with the grading of recommendations assessment, development, and evaluation (GRADE) approach (Tables 3 and 4). An overview for the outcomes of the main analysis and for the two subgroup analyses are provided in Tables 3 and 4. The outcomes of the main analysis ranged from very low to low largely due to study designs of included studies (retrospective) and study biases. The certainty of the evidence for outcomes for histologically confirmed NAFLD versus control also ranged from low to very low due to study design, risk of bias, and imprecision. Due to a lack of studies, perioperative mortality could not be evaluated.

\section{Discussion}

The global prevalence of the metabolic syndrome is estimated at a quarter of the world's population. NAFLD affects more than 200 million people worldwide and approximately $20 \%$ progress further to NASH [35]. These conditions not only increase primary liver cancer incidences but are a major risk factor for other cancers, such as colorectal, which often metastasizes to the liver $[3,4]$. With more than 1.9 million new cases of colorectal cancers per year, $70 \%$ with hepatic metastases, and 0.9 million annual new cases of liver cancer, liver surgery remains the preferred treatment for most hepatic lesions $[36,37]$.

The armamentarium of hepatobiliary surgery is continuously expanding, thus widening the indications for surgery as well $[38,39]$. With constant evolution of the field, it is necessary to re-evaluate accepted dogmas with 
Table 3 Certainty of the evidence for outcomes of the main analysis

\begin{tabular}{|c|c|c|c|}
\hline Outcome & № of included studies & Certainty of the evidence (GRADE) & $\begin{array}{l}\text { Relative effect } \\
(95 \% \mathrm{Cl})\end{array}$ \\
\hline Overall complications & 11 (retrospective) & $\begin{array}{l}\oplus \oplus \circ O \\
\text { LOW }\end{array}$ & $\begin{array}{l}\text { OR } 1.55 \\
{[1.05 ; 2.29]}\end{array}$ \\
\hline Major complications & 8 (retrospective) & $\begin{array}{l}\oplus \oplus \circ \circ \\
\text { LOW }\end{array}$ & $\begin{array}{l}\text { OR } 1.97 \\
{[1.13 ; 3.43]}\end{array}$ \\
\hline Minor complications & 8 (retrospective) & $\begin{array}{l}\oplus O O O \\
\text { VERY LOW }\end{array}$ & $\begin{array}{l}\text { OR } 0.72 \\
{[0.41 ; 1.29]}\end{array}$ \\
\hline PHLF & 6 (retrospective) & $\begin{array}{l}\text { ๑OOO } \\
\text { VERY LOW }\end{array}$ & $\begin{array}{l}\text { OR } 1.83 \\
{[0.86 ; 3.91]}\end{array}$ \\
\hline Postoperative biliary leakage & 8 (retrospective) & $\begin{array}{l}\oplus \oplus \circ O \\
\text { LOW }\end{array}$ & $\begin{array}{l}\text { OR } 1.43 \\
{[0.77 ; 2.63]}\end{array}$ \\
\hline Postoperative hemorrhage & 5 (retrospective) & $\begin{array}{l}\oplus \oplus \circ O \\
\text { LOW }\end{array}$ & $\begin{array}{l}\text { OR } 1.76 \\
{[1.23 ; 2.50]}\end{array}$ \\
\hline Postoperative infection & 8 (retrospective) & $\begin{array}{l}\oplus \oplus \circ O \\
\text { LOW }\end{array}$ & $\begin{array}{l}\text { OR } 1.63 \\
{[1.03 ; 2.57]}\end{array}$ \\
\hline Perioperative mortality & 2 (retrospective) & $\begin{array}{l}\oplus O O O \\
\text { VERY LOW }\end{array}$ & $\begin{array}{l}\text { OR 4.84 } \\
\text { [0.00; 41157788.03] }\end{array}$ \\
\hline 90-days mortality & 5 (retrospective) & $\begin{array}{l}\oplus \oplus \circ O \\
\text { LOW }\end{array}$ & $\begin{array}{l}\text { OR } 1.55 \\
{[0.76 ; 3.15]}\end{array}$ \\
\hline 1-year overall survival & 5 (retrospective) & $\begin{array}{l}\oplus \oplus \circ O \\
\text { LOW }\end{array}$ & $\begin{array}{l}\text { OR } 0.83 \\
{[0.32 ; 2.14]}\end{array}$ \\
\hline 5-years overall survival & 6 (retrospective) & $\begin{array}{l}\oplus \oplus \circ O \\
\text { LOW }\end{array}$ & $\begin{array}{l}\text { OR } 1.07 \\
{[0.79 ; 1.45]}\end{array}$ \\
\hline Recurrence & 4 (retrospective) & $\begin{array}{l}\oplus \oplus \circ O \\
\text { LOW }\end{array}$ & $\begin{array}{l}\text { OR 1.06 } \\
{[0.87 ; 1.30]}\end{array}$ \\
\hline 1-year recurrence-free survival & 4 (retrospective) & $\begin{array}{l}\oplus \oplus \circ O \\
\text { LOW }\end{array}$ & $\begin{array}{l}\text { OR } 0.95 \\
{[0.47 ; 1.92]}\end{array}$ \\
\hline 5-year recurrence-free survival & 5 (retrospective) & $\begin{array}{l}\oplus \oplus \circ O \\
\text { LOW }\end{array}$ & $\begin{array}{l}\text { OR } 1.20 \\
{[0.91 ; 1.59]}\end{array}$ \\
\hline
\end{tabular}

Cl confidence interval, $O R$ odds ratio

current treatment possibilities and test the boundaries of presumably known risk factors. Experienced hepatobiliary surgeons must be included in tumor boards and treatment discussions to offer more patients potentially curative options within the realm of current possibilities [40].

Despite numerous clinical scores for NAFLD/MAFLD, a reliable clinical assessment for patients with manifesting MAFLD is not yet available [41]. Liquid-based biopsies have varying reliability with most investigated factors associated with steatohepatitis or fibrosis, meaning already established NASH [41]. The need for adequate and reliable preoperative assessments is evident, to identify patients in different stages of MAFLD and, thus, structure individualized therapy plans. This need is also underlined by the results of this meta-analysis. Patients with the MetS were not at significantly higher risk for PHLF, while patients with histologically confirmed NAFLD were. The question remains, at what stage the risk of PHLF increase in patients suffering from the MetS. Although postoperative hemorrhage presents a risk in liver surgery, this has not previously been described as a specific complication for the MetS. However, the significant increase of postoperative bleeding is evident in this meta-analysis. As the liver is the major source of mature pro- and anticoagulative factors and the balance of these is upset after hepatectomies [42], investigating individual coagulation profiles of patients with and without the MetS subjected to liver surgery becomes essential.

The recurrence rates for resected tumors were similar in patients suffering from MAFLD versus other etiologies, indicating that this group too profits from surgery. Additionally, survival rates were similar to controls in the main and subgroup analyses, further solidifying this conclusion.

This is the first meta-analysis to investigate the effect of MetS on hepatic resection and chosen endpoints are of direct relevance for surgery and oncological assessment. The limitation of this meta-analysis is primarily rooted in the publications that met the criteria for inclusion. As most studies were retrospective and investigated unmatched cohorts, there is a risk of bias. Patients with the MetS and those with histopathologically confirmed 
Table 4 Certainty of evidence for outcomes of the subgroup analysis for histologically confirmed NAFLD versus control

\begin{tabular}{|c|c|c|c|}
\hline Outcome & № of included studies & Certainty of the evidence (GRADE) & $\begin{array}{l}\text { Relative effect } \\
(95 \% \mathrm{Cl})\end{array}$ \\
\hline Overall complications & 5 (retrospective) & $\begin{array}{l}\oplus \oplus \circ O \\
\text { LOW }\end{array}$ & $\begin{array}{l}\text { OR } 1.86 \\
{[0.84 ; 4.11]}\end{array}$ \\
\hline Major complications & 4 (retrospective) & $\begin{array}{l}\oplus \oplus O O \\
\text { LOW }\end{array}$ & $\begin{array}{l}\text { OR 2.46 } \\
{[0.60 ; 10.06]}\end{array}$ \\
\hline Minor complications & 4 (retrospective) & $\begin{array}{l}\oplus O O O \\
\text { VERY LOW }\end{array}$ & $\begin{array}{l}\text { OR } 0.69 \\
{[0.11 ; 4.35]}\end{array}$ \\
\hline PHLF & 2 (retrospective) & $\begin{array}{l}\oplus \oplus \circ \circ \\
\text { LOW }\end{array}$ & $\begin{array}{l}\text { OR } 4.87 \\
{[1.22 ; 19.47]}\end{array}$ \\
\hline Postoperative biliary leakage & 3 (retrospective) & $\begin{array}{l}\oplus O O O \\
\text { VERY LOW }\end{array}$ & $\begin{array}{l}\text { OR } 1.21 \\
{[0.01 ; 178.13]}\end{array}$ \\
\hline Postoperative hemorrhage & 3 (retrospective) & $\begin{array}{l}\oplus \oplus O O \\
\text { LOW }\end{array}$ & $\begin{array}{l}\text { OR 2.08 } \\
{[0.49 ; 8.85]}\end{array}$ \\
\hline Postoperative infection & 2 (retrospective) & $\begin{array}{l}\oplus O O O \\
\text { VERY LOW }\end{array}$ & $\begin{array}{l}\text { OR 2.17 } \\
\text { [0.00; 5413.25] }\end{array}$ \\
\hline Perioperative mortality & 0 & $\mathrm{NE}$ & $\mathrm{NE}$ \\
\hline 90-days mortality & 2 (retrospective) & $\begin{array}{l}\oplus O O O \\
\text { VERY LOW }\end{array}$ & $\begin{array}{l}\text { OR 1.90 } \\
{[0.00 ; 179526.98]}\end{array}$ \\
\hline 1-year overall survival & 2 (retrospective) & $\begin{array}{l}\text { ๑OOO } \\
\text { VERY LOW }\end{array}$ & $\begin{array}{l}\text { OR } 0.79 \\
{[0.00 ; 93283.03]}\end{array}$ \\
\hline 5-years overall survival & 3 (retrospective) & $\begin{array}{l}\oplus \oplus O O \\
\text { LOW }\end{array}$ & $\begin{array}{l}\text { OR } 1.39 \\
{[0.85 ; 2.26]}\end{array}$ \\
\hline Recurrence & 3 (retrospective) & $\begin{array}{l}\oplus \oplus O O \\
\text { LOW }\end{array}$ & $\begin{array}{l}\text { OR } 1.11 \\
{[0.69 ; 1.79]}\end{array}$ \\
\hline 1-year recurrence-free survival & 2 (retrospective) & $\begin{array}{l}\oplus \oplus O O \\
\text { LOW }\end{array}$ & $\begin{array}{l}\text { OR } 0.96 \\
{[0.00 ; 920.79]}\end{array}$ \\
\hline 5-year recurrence-free survival & 3 (retrospective) & $\begin{array}{l}\oplus \oplus O O \\
\text { LOW }\end{array}$ & $\begin{array}{l}\text { OR } 1.23 \\
{[0.76 ; 1.99]}\end{array}$ \\
\hline
\end{tabular}

Cl confidence interval, OR odds ratio, NE not evaluated

NAFLD are considered poor candidates due to comorbidities and hepatic changes-this may lead to a selection bias. Additionally, included studies used different criteria for diagnosis of MetS-histological confirmation of MetS-associated changes in the liver, two or three criteria for MetS as defined by national or international societies. Definition of NAFLD also varied between studies with several studies utilizing the NASH Clinical Research Network criteria, some studies choosing SAF score and some opting for criteria proposed by the national association for the study of liver disease [32-34]. This inhomogeneity of definition harbors a potential bias and underlines the need for systematic studies in the area and consensus of definitions. Comparative controls also differed between groups: intrahepatic cholangiocarcinomas were compared in patients with and without the MetS, as were various benign and malignant lesions without further division. Patients with MetS-associated HCC were compared to various etiologies, hepatitis Cor B- -associated HCC, hepatitis without further subdivision, and alcohol-associated HCC. Thus, the overall control group consists of a clinically representative albeit heterogenic group with sufficiently high power.
Although heterogeneity of included studies was generally acceptable and often low in subgroup analyses, these analyses were often based on three studies alone. This inevitably leads to a wider 95\% CI and, thus, deficit of significance. Unarguably, more studies investigating different stages of MAFLD, from simple MetS to manifesting NASH will strengthen the certainty of evidence provided by this meta-analysis. Especially impact of metabolically associated steatohepatitis on outcomes after hepatectomy will provide invaluable insight.

More emphasis should be placed on reliable but noninvasive markers of MAFLD-stages. Although a liver biopsy can provide valuable information and is the current standard of assessment for NAFLD/NASH diagnosis, due to associated risks and limited clinical relevance in absence of a tumor burden, this intervention has lost its popularity [43]. Additionally, the biopsy only assesses a minuscule area of the liver, assuming validity in generalization. When a resection of a liver tumor is planned, a preoperatively diagnosed MAFLD would not lead to an alteration of the treatment plan, thus a biopsy could be combined with a resection, in which case a representative area can be obtained for histopathological assessment. A serum-based marker would 
present a superior test to assess the patient, as little infrastructure and less specialized personnel are required. Also, a serum-based marker could be offered to a larger population. Additionally, the need for an early and reliable PHLF marker is evident [8]. As PHLF remains the most feared complication after hepatectomy with rates reaching up to $30 \%$, early detection is invaluable to provide patients with adequate care and evade lethal consequences [7]. Liver systems medicine approach and multidisciplinary consortia that place their emphasis on PHLF and MAFLD may unveil intimate knowledge on pathogenesis and suggest viable treatment options that are currently lacking (https://www. lisym.org/).

\section{Conclusion}

The metabolic syndrome presents a risk factor in hepatic surgery with patients suffering significantly more complications. Yet, liver-specific complications, such as PHLF and biliary leakage rates are not increased. Patients with MetS are at higher risk for postoperative hemorrhage and infections - complications that can be managed through adjustment of the surgical technique and perioperative care. Improvement of the preoperative differentiation of MetS with and without hepatic manifestation is overdue, as patients with MetS-associated histological changes are at greater risk for PHLF. Developing prognostic markers for PHLF remains a priority. Despite higher PHLF rates, patients with MAFLD show similar 1- and 5-year overall and recurrence-free survivals, thus clearly benefiting from surgery as much as patients with liver malignancies of other etiologies.

\section{Abbreviations \\ FLR: Future liver remnant; GRADE: Grading of recommendations assessment, development and evaluation; HCC: Hepatocellular carcinoma; MAFL D: Metabolic-associated fatty liver disease; MetS: Metabolic syndrome; NAFL D: Non-alcoholic fatty liver disease; NASH: Non-alcoholic steatohepatitis; OR: Odds ratio; OS: Overall survival; PHLF: Post-hepatectomy liver failure; QUIPS: Quality in Prognostic Studies; RFS: Recurrence-free survival; SAF score: steatosis, activity, fibrosis score}

\section{Supplementary Information}

The online version contains supplementary material available at https://doi. org/10.1186/s12916-022-02239-x.

Additional file 1. Supplementary material.

\section{Acknowledgements}

Not applicable.

\section{Authors' contributions}

AML conceived the original idea. EK performed the literature search. AML and JF performed the systematic review of publications and data extraction. $A M L, K H, J F, S F, R K$, and PP developed the methodology. AML, JF, and MT performed the statistical analyses. AML drafted the manuscript. $A M L, K H, J F$, SF, ES, MT, RK, RK, AB, and PP revised and edited the manuscript. All authors read and approved the final manuscript.

\section{Funding}

The authors have not declared a specific grant for this research from any funding agency in the public, commercial or not-for-profit sectors. Open Access funding enabled and organized by Projekt DEAL.

\section{Availability of data and materials}

All data generated or analyzed during this study are included in this published article and its supplementary information files.

\section{Declarations}

Ethics approval and consent to participate

Not applicable.

\section{Consent for publication}

Not applicable.

\section{Competing interests}

The authors declare that they have no competing interests.

\section{Author details}

${ }^{1}$ Department of General, Visceral, and Transplantation Surgery, Heidelberg University Hospital, Heidelberg, Germany. ${ }^{2}$ The Study Center of the German Surgical Society (SDGC), Heidelberg University Hospital, Heidelberg, Germany. ${ }^{3}$ Department of Surgery, Cantonal Hospital Thurgau, Frauenfeld, Switzerland.

Received: 20 September 2021 Accepted: 6 January 2022

Published online: 28 January 2022

\section{References}

1. Saklayen MG. The Global Epidemic of the Metabolic Syndrome. Curr Hypertens Rep. 2018;20(2):12. https://doi.org/10.1007/s11906-018-0812-z.

2. Shariq OA, Hanson KT, McKenna NP, Kelley SR, Dozois EJ, Lightner AL, et al. Does Metabolic Syndrome Increase the Risk of Postoperative Complications in Patients Undergoing Colorectal Cancer Surgery? Dis Colon Rectum. 2019; 62(7):849-58. https://doi.org/10.1097/DCR.0000000000001334.

3. Esposito K, Chiodini P, Colao A, Lenzi A, Giugliano D. Metabolic syndrome and risk of cancer: a systematic review and meta-analysis. Diabetes Care. 2012;35(11):2402-11. https://doi.org/10.2337/dc12-0336.

4. Welzel TM, Graubard BI, Zeuzem S, El-Serag HB, Davila JA, McGlynn KA. Metabolic syndrome increases the risk of primary liver cancer in the United States: a study in the SEER-Medicare database. Hepatology (Baltimore, Md). 2011;54(2):463-71.

5. Eslam M, Sanyal AJ, George J. MAFLD: A Consensus-Driven Proposed Nomenclature for Metabolic Associated Fatty Liver Disease. Gastroenterology. 2020;158(7):1999-2014.e1.

6. Perumpail RB, Wong RJ, Ahmed A, Harrison SA. Hepatocellular Carcinoma in the Setting of Non-cirrhotic Nonalcoholic Fatty Liver Disease and the Metabolic Syndrome: US Experience. Dig Dis Sci. 2015;60(10):3142-8. https:// doi.org/10.1007/s10620-015-3821-7.

7. Kauffmann R, Fong Y. Post-hepatectomy liver failure. Hepatobiliary Surg Nutr. 2014;3(5):238-46. https://doi.org/10.3978/j.issn.2304-3881.2014.09.01.

8. Murtha-Lemekhova A, Fuchs J, Ghamarnejad O, Nikdad M, Probst P, Hoffmann K. Influence of cytokines, circulating markers and growth factors on liver regeneration and post-hepatectomy liver failure: a systematic review and meta-analysis. Sci Rep. 2021;11(1):13739. https://doi.org/10.1038/ s41598-021-92888-4.

9. Asencio JM, García Sabrido JL, Olmedilla L. How to expand the safe limits in hepatic resections? J Hepato-biliary-pancreatic Sci. 2014;21(6):399-404. https://doi.org/10.1002/jhbp.97.

10. Page MJ, McKenzie JE, Bossuyt PM, Boutron I, Hoffmann TC, Mulrow CD, et al. The PRISMA 2020 statement: An updated guideline for reporting systematic reviews. Int J Surg (Lond Engl). 2021:88:105906.

11. Higgins JPT TJ, Chandler J, Cumpston M, Li T, Page MJ, Welch VA. Cochrane Handbook for Systematic Reviews of Interventions version 6.2 (updated February 2021). Cochrane. 2021. Available from www.training.cochrane.org/ handbookURL. Accessed 23 Aug 2021.

12. Anastasia Lemekhova JF, Feiler S, Klotz R, Kalkum E, Probst P, Hoffmann K. Influence of metabolic associated steatosis on liver function, regeneration and PHLF after hepatectomy: a systematic review and meta-analysis. In: PROSPERO; 2021. CRD42021253768 Available from: https://www.crd.york.ac. 
uk/prospero/display_record.php?ID=CRD42021253768. Accessed 15 Aug 2021.

13. Kalkum E, Klotz R, Seide S, Hüttner FJ, Kowalewski K-F, Nickel F, et al. Systematic reviews in surgery-recommendations from the Study Center of the German Society of Surgery. Langenbecks Arch Surg. 2021;406(6):172331. https://doi.org/10.1007/s00423-021-02204-x.

14. Hayden JA, van der Windt DA, Cartwright JL, Côté P, Bombardier C. Assessing bias in studies of prognostic factors. Ann Intern Med. 2013;158(4): 280-6. https://doi.org/10.7326/0003-4819-158-4-201302190-00009.

15. Foroutan F, Guyatt G, Zuk V, Vandvik PO, Alba AC, Mustafa R, et al. GRADE Guidelines 28: Use of GRADE for the assessment of evidence about prognostic factors: rating certainty in identification of groups of patients with different absolute risks. J Clin Epidemiol. 2020;121:62-70. https://doi. org/10.1016/j.jclinepi.2019.12.023.

16. HBJ S, Guyatt G, Oxman A. GRADE handbook for grading quality of evidence and strength of recommendations. Updated October 2013. In: The GRADE Working Group; 2013. Available from guidelinedevelopment.org/ha ndbook. Accessed 15 Aug 2021.

17. Billeter AT, Müller PC, Albrecht T, Roessler S, Löffler M, Lemekhova A, et al. Impact of Type 2 Diabetes on Oncologic Outcomes of Hepatocellular Carcinomas in Non-Cirrhotic, Non-alcoholic Steatohepatitis: a Matched-Pair Analysis. J Gastrointest Surg. 2020;25(5):1193-202.

18. Jung YB, Yoo JE, Han DH, Kim KS, Choi JS, Kim DY, et al. Clinical and survival outcomes after hepatectomy in patients with non-alcoholic fatty liver and hepatitis B-related hepatocellular carcinoma. HPB. 2020;23(7):1113-22.

19. Koh YX, Tan HJ, Liew YX, Syn N, Teo JY, Lee SY, et al. Liver Resection for Nonalcoholic Fatty Liver Disease-Associated Hepatocellular Carcinoma. J Am Coll Surg. 2019;229(5):467-78.e1.

20. Pais R, Fartoux L, Goumard C, Scatton O, Wendum D, Rosmorduc O, et al. Temporal trends, clinical patterns and outcomes of NAFLD-related HCC in patients undergoing liver resection over a 20-year period. Aliment Pharmacol Ther. 2017;46(9):856-63. https://doi.org/10.1111/apt.14261.

21. Tian YH, Li T, Qi SL, Alhourani H, Luo BY, Chenqin J, et al. The impact of metabolic syndrome (MetS) on surgical outcome for patients with mostly HBV-related hepatocellular carcinoma (HCC) underwent hepatectomy. J Surg Oncol. 2020;122(4):699-706. https://doi.org/10.1002/jso.26055.

22. Tian YH, Lyu H, He YH, Xia Y, Li J, Shen F. Comparison of Hepatectomy for Patients with Metabolic Syndrome-Related HCC and HBV-Related HCC. J Gastrointest Surg. 2018;22(4):615-23. https://doi.org/10.1007/s11 605-017-3629-1.

23. Wakai T, Shirai Y, Sakata J, Korita PV, Ajioka Y, Hatakeyama K. Surgical outcomes for hepatocellular carcinoma in nonalcoholic fatty liver disease. J Gastrointest Surg. 2011;15(8):1450-8. https://doi.org/10.1007/ s1 1605-011-1540-8.

24. Yang T, Hu LY, Li ZL, Liu K, Wu H, Xing H, et al. Liver Resection for Hepatocellular Carcinoma in Non-alcoholic Fatty Liver Disease: a Multicenter Propensity Matching Analysis with HBV-HCC. J Gastrointest Surg. 2020;24(2): 320-9. https://doi.org/10.1007/s11605-018-04071-2.

25. Yoshida N, Takayama T, Midorikawa Y, Higaki T, Nakayama H, Moriguchi M, et al. Surgical Outcomes in Patients with Hepatocellular Carcinoma Associated with Metabolic Syndrome. World J Surg. 2015;39(2):471-7. https://doi.org/10.1007/s00268-014-2828-0.

26. Conci S, Cipriani F, Donadon M, Marchitelli I, Ardito F, Famularo S, et al. Hepatectomy for Metabolic Associated Fatty Liver Disease (MAFLD) related HCC: Propensity case-matched analysis with viral- and alcohol-related HCC. Eur J Surg Oncol. 2021. https://doi.org/10.1016/j.ejso.2021.07.015.

27. Hobeika C, Cauchy F, Poté N, Rautou PE, Durand F, Farges O, et al. Shortand Long-Term Outcomes of Liver Resection for Intrahepatic Cholangiocarcinoma Associated with the Metabolic Syndrome. World J Surg. 2019;43(8):2048-60. https://doi.org/10.1007/s00268-019-04996-y.

28. Nishioka T, Kubo S, Tanaka S, Wakasa K, Takemura S, Kinoshita M, et al. Outcomes of Hepatic Resection in Intrahepatic Cholangiocarcinoma Patients with Diabetes, Hypertension, and Dyslipidemia: Significance of Routine Follow-Up. Liver Cancer. 2016;5(2):107-20. https://doi.org/10.1159/0003 67752.

29. Bhayani NH, Hyder O, Frederick W, Schulick RD, Wolgang CL, Hirose K, et al. Effect of metabolic syndrome on perioperative outcomes after liver surgery: A National Surgical Quality Improvement Program (NSQIP) analysis. Surgery. 2012;152(2):218-26. https://doi.org/10.1016/j.surg.2012.05.037.

30. Zarzavadjian Le Bian A, Costi R, Constantinides V, Smadja C. Metabolic disorders, non-alcoholic fatty liver disease and major liver resection: an underestimated perioperative risk. J Gastrointest Surg. 2012;16(12):2247-55. https://doi.org/10.1007/s11605-012-2044-x.

31. Fagenson AM, Pitt HA, Moten AS, Karhadkar SS, Di Carlo A, Lau KN. Fatty liver: The metabolic syndrome increases major hepatectomy mortality. Surgery. 2021;169(5):1054-60. https://doi.org/10.1016/j.surg.2020.11.021.

32. Kleiner DE, Brunt EM. Nonalcoholic Fatty Liver Disease: Pathologic Patterns and Biopsy Evaluation in Clinical Research. Semin Liver Dis. 2012;32(1):3-13. https://doi.org/10.1055/s-0032-1306421.

33. Chalasani N, Younossi Z, Lavine JE, Diehl AM, Brunt EM, Cusi K, et al. The diagnosis and management of non-alcoholic fatty liver disease: Practice Guideline by the American Association for the Study of Liver Diseases, American College of Gastroenterology, and the American Gastroenterological Association. Hepatology (Baltimore, Md). 2012;55(6): 2005-23.

34. Bedossa P, Poitou C, Veyrie N, Bouillot J-L, Basdevant A, Paradis V, et al. Histopathological algorithm and scoring system for evaluation of liver lesions in morbidly obese patients. Hepatology (Baltimore, Md). 2012;56(5): $1751-9$.

35. Pfister D, Núñez NG, Pinyol R, Govaere O, Pinter M, Szydlowska M, et al. NASH limits anti-tumour surveillance in immunotherapy-treated HCC. Nature. 2021;592(7854):450-6. https://doi.org/10.1038/s41586-021-03362-0.

36. Sung H, Ferlay J, Siegel RL, Laversanne M, Soerjomataram I, Jemal A, et al. Global Cancer Statistics 2020: GLOBOCAN Estimates of Incidence and Mortality Worldwide for 36 Cancers in 185 Countries. CA: A Cancer J Clin. 2021;71(3):209-49. https://doi.org/10.3322/caac.21660.

37. Fowler KJ, Linehan DC, Menias CO. Colorectal Liver Metastases: State of the Art Imaging. Ann Surg Oncol. 2013;20(4):1185-93. https://doi.org/10.1245/s1 0434-012-2730-7.

38. Murtha-Lemekhova A, Fuchs J, Schulz E, Sterkenburg A, Probst P, Hoffmann $K$. Pushing the limit of liver regeneration - Safety and survival after monosegment-ALPPS: systematic review and individual patient data metaanalysis. HPB (Oxford). 2021. https://doi.org/10.1016/j.hpb.2021.06.427.

39. Schnitzbauer AA, Lang SA, Goessmann H, Nadalin S, Baumgart J, Farkas SA, et al. Right portal vein ligation combined with in situ splitting induces rapid left lateral liver lobe hypertrophy enabling 2-staged extended right hepatic resection in small-for-size settings. Ann Surg. 2012;255(3):405-14. https://doi. org/10.1097/SLA.0b013e31824856f5.

40. Vega EA, Salehi O, Nicolaescu D, Dussom EM, Alarcon SV, Kozyreva O, et al. Failure to Cure Patients with Colorectal Liver Metastases: The Impact of the Liver Surgeon. Ann Surg Oncol. 2021;28(12):7698-706.

41. Vilar-Gomez E, Chalasani N. Non-invasive assessment of non-alcoholic fatty liver disease: Clinical prediction rules and blood-based biomarkers. J Hepatol. 2018;68(2):305-15. https://doi.org/10.1016/j.jhep.2017.11.013.

42. Mallett SV, Sugavanam A, Krzanicki DA, Patel S, Broomhead RH, Davidson $B R$, et al. Alterations in coagulation following major liver resection. Anaesthesia. 2016;71(6):657-68. https://doi.org/10.1111/anae.13459.

43. Russo FP, Imondi A, Lynch EN, Farinati F. When and how should we perform a biopsy for HCC in patients with liver cirrhosis in 2018? A review. Dig Liver Dis. 2018;50(7):640-6. https://doi.org/10.1016/j.dld.2018.03.014.

\section{Publisher's Note}

Springer Nature remains neutral with regard to jurisdictional claims in published maps and institutional affiliations.

Ready to submit your research? Choose BMC and benefit from:

- fast, convenient online submission

- thorough peer review by experienced researchers in your field

- rapid publication on acceptance

- support for research data, including large and complex data types

- gold Open Access which fosters wider collaboration and increased citations

- maximum visibility for your research: over $100 \mathrm{M}$ website views per year

At $\mathrm{BMC}$, research is always in progress.

Learn more biomedcentral.com/submission 